\title{
Acute gastric volvulus
}

\author{
Abdul Hai · Sanjay Goel $\cdot$ Saqib Shahab $\cdot$ \\ Sudesh Kumar • Vishal Sharma
}

Received: 10 June 2010/Accepted: 1 July 2010/Published online: 20 July 2010

(C) SIMI 2010

An 18-year-old man presented with complaints of sudden onset pain in the epigastrium radiating to the left lower chest, starting 7 days prior followed by non-productive and persistent retching. On examination, the patient had a tachycardia (pulse rate 108 beats $/ \mathrm{min}$ ) and a blood pressure of 102/66 mmHg. His respiratory rate was 22 breaths $/ \mathrm{min}$ and was afebrile. On chest examination, air entry was markedly reduced on the left side. The abdomen was not distended; an ovoid-shaped tender organ was felt in the epigastrium. The rest of the abdominal examination was normal. A single gentle attempt of nasogastric tube insertion was done, but insertion beyond the first mark was not possible. A plain X-ray study of the chest and abdomen done earlier showed a double bubble sign (Fig. 1), Contrast films showed a dilatated and tortous dumbbell-shaped stomach overlapping the left lower chest (Fig. 2). Although a computed tomography (CT) scan of the abdomen would have been preferable, the barium study had been done before the patient was referred to us [1].

In view of these findings a diagnosis of gastric volvulus with herniation into the left chest cavity was made, and the patient was urgently operated upon after appropriate preparation. The operative findings revealed that the left lobe of the liver and spleen were shifted medially and

A. Hai $\cdot$ S. Goel $\cdot$ S. Shahab $\cdot$ S. Kumar

Department of Surgery, University College of Medical Sciences and Associated GTB Hospital, University of Delhi, Delhi, India

V. Sharma

Department of Medicine, University College of Medical

Sciences and Associated GTB Hospital,

University of Delhi, Delhi, India

V. Sharma $(\bowtie)$

1819 Gobind Nagar Chheharta, Amritsar, Punjab, India

e-mail: docvishalsharma@gmail.com downwards so that the spleen was lying in the epigastrium (Fig. 3). The stomach was largely missing from the abdomen, and only a small pouch of fundus was visible just below the spleen. The entire distal stomach had herniated through a defect in the diaphragm posterolaterally on the left side. On needle aspiration about $500 \mathrm{ml}$ of barium mixed fluid came out from the stomach pouch, but the stomach still refused to come down into the abdomen. So a thoracotomy was performed by extending the upper end of the laparotomy incision into the left side of the chest and the diaphragm was incised up to the defect posteriorly. The stomach was reduced into the abdominal cavity. It was viable, but inflammed and ballooned out with rotation of $>180^{\circ}$ anticlockwise (Fig. 4). It was derotated, and emptied by Ryle's tube aspiration. A gastropexy was done. The defect in the diaphragm was repaired with nonabsorbable sutures, and all the incisions were closed after placing a thoracostomy tube to drainage. The patient improved in the postoperative period, and was discharged on the seventh postoperative day.

The normal stomach is stabilised and prevented from twisting into a volvulus by the ligamentous supports, i.e. the gastrohepatic, gastrocolic, oesophagophrenic and gastrosplenic ligaments. The relative fixity of the pylorus and gastro-oesophageal junction also helps to maintain the normal position of the stomach. A volvulus can occur when these attachments are lax or absent. It may also occur secondary to adjoining structural defects like congenital diaphragmatic hernia, hiatus hernia, eventration of diaphragm, paraoesophageal hernia, splenomegaly, or gastric outlet obstruction [2]. Gastric volvulus is classified into three types: organoaxial: the stomach rotates around a longitudinal axis between the gastro-esophageal junction and the pylorus. This is the most common type occurring in approximately $59 \%$ of cases, and is usually associated with 


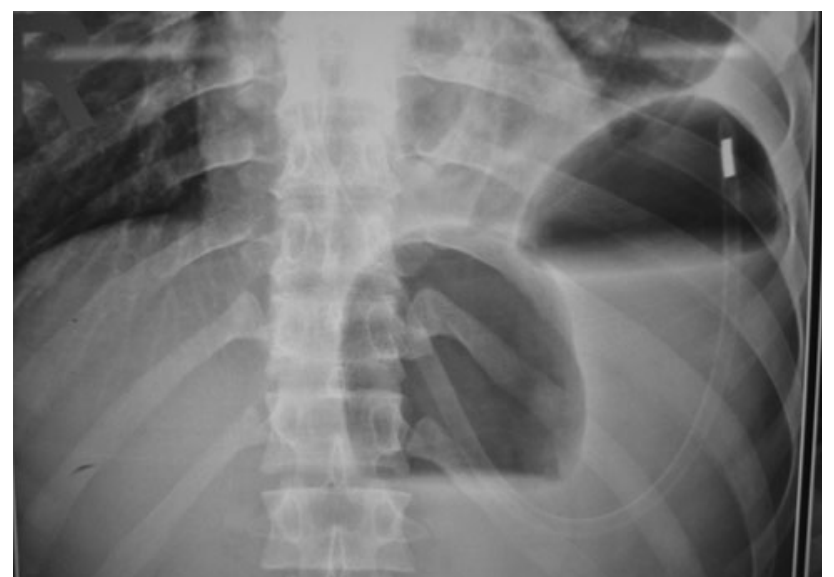

Fig. 1 Plane X-ray shows double bubble sign, one bubble above and the other below the left dome of diaphragm

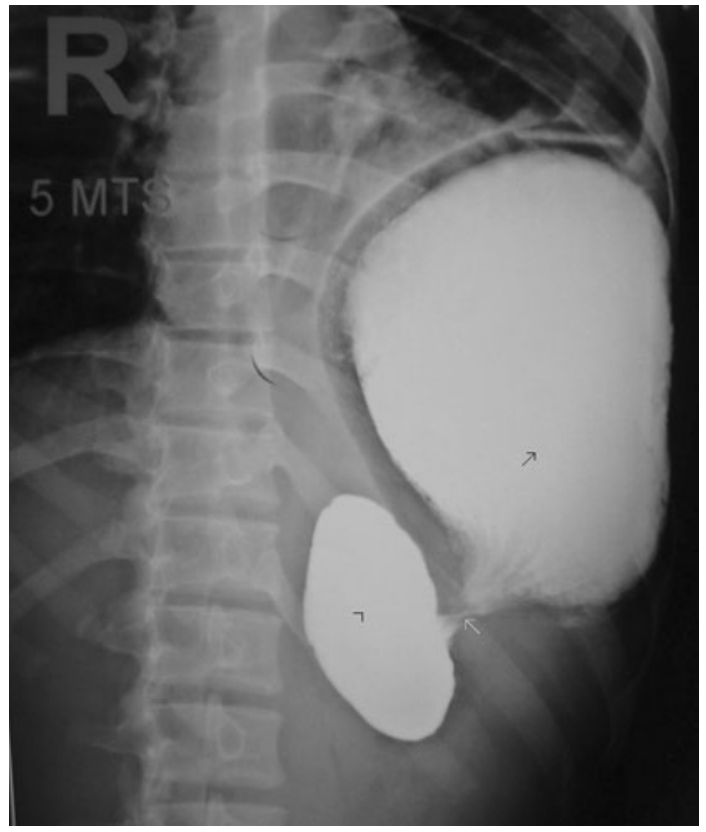

Fig. 2 Barium contrast study showing a dumb-bell shaped stomach. There is a halo around the upper pouch because of edema (black arrowhead the fundus of the stomach, black arrow the intrathoracic body of stomach, white arrow the constriction at the level of the diaphragm)

diaphragmatic defects. It is common in children particularly below 1 year of age. Mesentericoaxial: the stomach rotates around an axis running between the lesser and greater curvatures. The position of the stomach becomes upside down [3]. Patients with this type usually have no diaphragmatic defects. Mixed type: the stomach rotates with a mix of the above two patterns. This is a very rare variety.

Acute gastric volvulus usually presents as sudden onset severe epigastric or left upper abdominal pain, which may

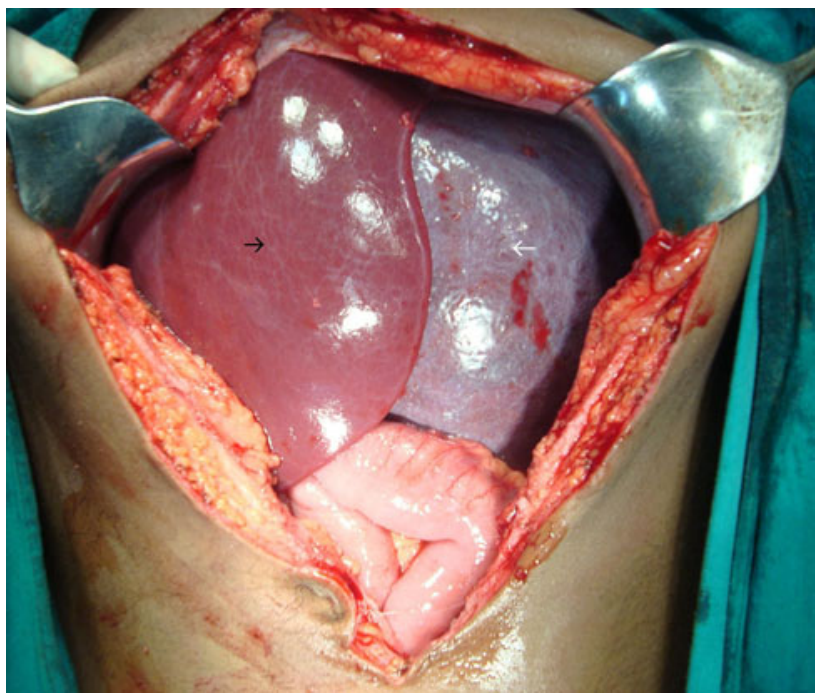

Fig. 3 Operative photograph showing the spleen and the left lobe of the liver shifted towards the right side and downwards. The stomach is conspicious by its absence (black arrow liver, white arrow spleen)

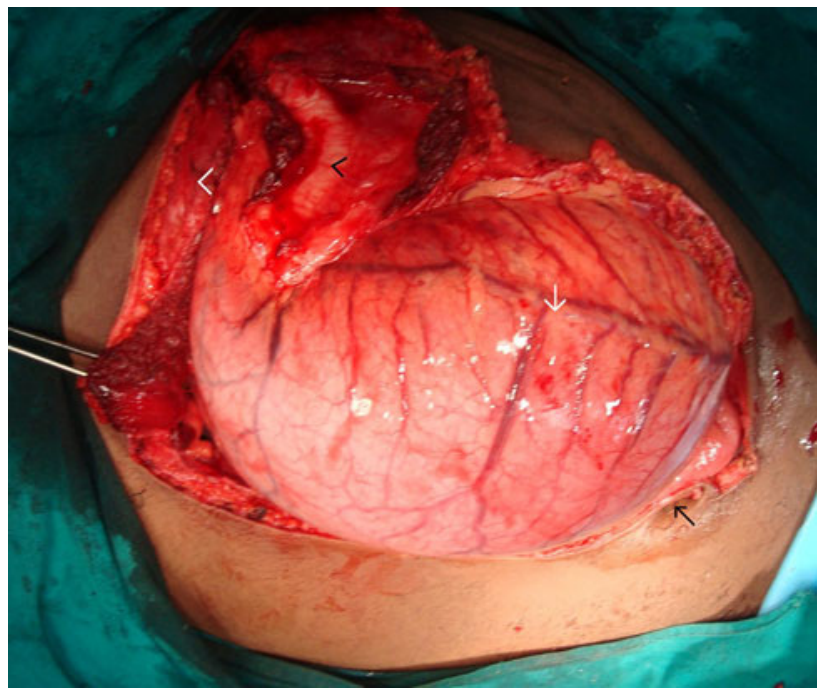

Fig. 4 Operative photograph showing a distended, inflammed and rotated stomach. The pylorus is stretched, lying above the body towards the thoracotomy incision (black arrow the umbilicus, white arrow the rotated stomach, black arrowhead the stretched pylorus, white arrowhead the thoracotomy incision)

be radiating to chest, neck, shoulder and back. It may mimic an acute cardiac event. There may be an initial episode of vomiting followed by progressive distension and nonproductive retching. Borchardt [4] described the classic clinical triad in gastric volvulus of severe epigastric pain, retching with no vomiting, and an inability to pass a nasogastric tube. There may be minimal abdominal signs in the case of intrathoracic stomach as in our patient. Patients suspected to be suffering from acute gastric volvulus on the basis of history and physical examination should be 
resuscitated with i.v. fluids, oxygen supplementation and electrolyte correction. A chest X-ray study may show a retrocardiac, gas-filled viscus with an air-fluid level. A plain abdominal X-ray study may reveal a hugely distended viscus in the upper abdomen, and absence of gas in the distal bowel. The treatment of an acute gastric volvulus is an emergency laparotomy alone, or combined with a thoracotomy when necessary. The viability of the stomach should be assessed. The treatment includes decompression, derotation and reduction of the stomach, and prevention of a recurrence by repair of the causative defects. Decompression by needle aspiration from the stomach pouch should initially be attempted, otherwise thoracotomy may be necessary, as we had to do. In patients with diaphragmatic defects, reduction with a repair may suffice [5]. In an intraabdominal stomach, gastropexy to the diaphragm and the anterior abdominal wall should be done. In patients who are poor surgical candidates, endoscopic reduction may be attempted.
Conflict of interest None.

\section{References}

1. Attene J, Pepe G, Vanni S, Chiarlone M, Dilaghi B, Grifoni S (2009) A 64-year-old man admitted to the emergency department with an unusual case of intestinal obstruction: decision-making in the emergency department. Intern Emerg Med 4:45-51

2. Karande TP, Oak SN, Karmarkar SJ, Kulkarni BK, Deshmukh SS (1997) Gastric volvulus in childhood. J Postgrad med 43:46-47

3. Milne LW, Hunter JJ, Anshus JS (1994) Gastric volvulus: two cases and a review of the literature. J Emerg Med 12:299-306

4. Borchardt M (1904) Aus pathologie und therapie des magenvolvulus. Arch Klin Chir 74:243

5. Chattopadhyay A, Vepakomma D, Prakash B, Kumar V (2005) Is gastropexy required for all cases of gastric volvulus in children? Int Surg 90:151-154 